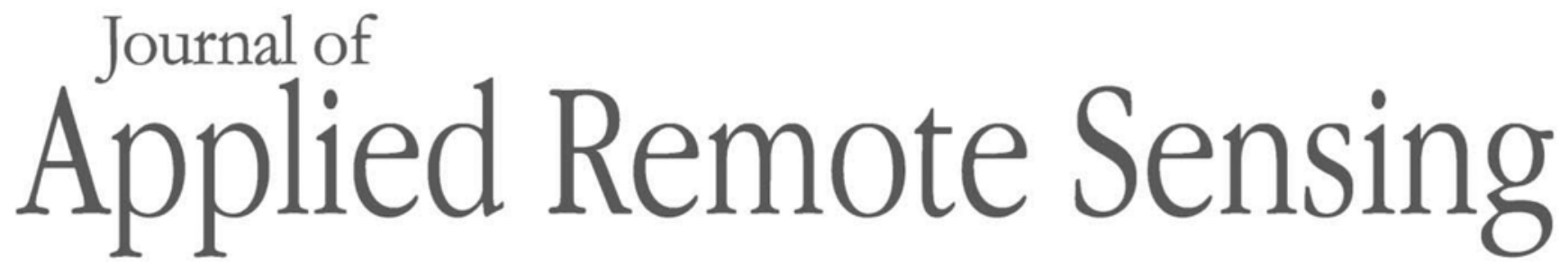

RemoteSensing.SPIEDigitalLibrary.org

\title{
Special Section Guest Editorial: Sparsity Driven High Dimensional Remote Sensing Image Processing and Analysis
}

\author{
Xin Huang \\ Paolo Gamba \\ Bormin Huang
}

Xin Huang, Paolo Gamba, Bormin Huang, "Special Section Guest Editorial: Sparsity Driven High 


\title{
Special Section Guest Editorial: Sparsity Driven High Dimensional Remote Sensing Image Processing and Analysis
}

\author{
Xin Huang, ${ }^{a}, *$ Paolo Gamba, ${ }^{b, *}$ and Bormin Huang ${ }^{c, *}$ \\ ${ }^{a}$ Wuhan University, School of Remote Sensing and Information Engineering, \\ \#129, Luoyu Road, Wuhan 430079, China \\ ${ }^{b}$ University of Pavia, Department of Electronics, Via Ferrata, 1, Pavia 27100, Italy \\ ${ }^{c}$ University of Wisconsin-Madison, Space Science and Engineering Center, \\ 1225 West Dayton Street, Madison, Wisconsin 53706, USA
}

We are witnessing a tremendous increase in the amount of Earth observation (EO) data, which opens a new era of high-dimensional remotely sensed data application. A diversity of sensors is capable of providing high spectral and spatial resolution imagery with multiple viewing angles, multiple sensors, and dense time series, forming a high-dimensional data space in spatiotemporal and spectral domains. At the same time, a number of state-of-the-art image processing techniques, e.g., multiple feature extraction and kernel trick, tend to generate a high-dimensional feature space to improve the accuracy of image interpretation. In this context, in pace with the rapid developments in remote sensors and image analysis techniques, high-dimensional data processing is becoming a common and challenging issue.

In recent years, signal sparsity has become a powerful and promising statistical modeling tool for high-dimensional image processing and analysis. The sparsity prior is well suited for remote sensing applications, since the puzzling obstacles, such as limited reference data, complicated sensor malfunctions, and poor atmospheric conditions, can be naturally modeled as sparse recovery process with promising solutions. However, there exists a great imbalance between the current status and the great potential for sparsity-based high-dimensional remote sensing image processing. Hence, there is an urgent demand to comprehensively reveal the role of sparsity in remote sensing scenes and develop effective sparsity driven processing techniques for such informative and advanced datasets.

In line with these comments, the ten papers in this special section deal with different research lines. There are four papers about sparse coding (SC) based remote sensing image interpretation. "Sparse coding-based correlation model for land-use scene classification in high-resolution remote-sensing images" by K. Qi et al. designs SC-based correlograms to discriminate landuse classes with a lower reconstruction error than the widely used k-means approach. " $\ell_{1.2}$-norm regularized nonnegative low-rank and sparse affinity graph for remote sensing image segmentation" by $\mathrm{S}$. Tian et al. includes $\ell_{1.2}$-norm regularization into the low-rank representation paradigm, to capture the intrinsic spatial manifold structure of the high-spatialresolution remote sensing data. Additionally, it exploits the advantage of low rank and sparse representation to improve the performance of graph nonnegative factorization segmentation. "Temperature and emissivity separation via sparse representation with thermal airborne hyperspectral" by $\mathrm{C}$. $\mathrm{Li}$ et al. imposes sparse regularization on the temperature and emissivity separation process by using the dictionary from Johns Hopkins University's spectral library as a prior, implementing a robust retrieval technique for noisy signal conditions. Finally, "Geometric correction method for linear array pushbroom infrared imagery using compressive sampling" by J. Chen et al. leverages the equivalent bias angles to approximate the influence of the errors in the imaging process and adopts a compressive sensing method, with sparse coding as a core factor, to recover the equivalent bias angle signals.

From a different perspective, four papers focus on the endogenous sparsity property of the remote sensing data, neatly investigated to improve the application performance. In "Identifying

\footnotetext{
*Address all correspondence to: Xin Huang, E-mail: xhuang @whu.edu.cn; Paolo Gamba, E-mail: paolo.gamba@ unipv.it; Bormin Huang, E-mail: bormin@ssec.wisc.edu

() 2016 Society of Photo-Optical Instrumentation Engineers (SPIE)
} 
relevant hyperspectral bands using boruta: a temporal analysis of water hyacinth biocontrol" by $\mathrm{N}$. Agjee et al., two band selection methods are combined with random forest classifier to detect the efficacy of a water hyacinth biocontrol agent, driven by a sparsity assumption of the relevant hyperspectral reflectance. Successfully keeping all relevant bands, it is believed that the boruta algorithm can be utilized to undertake the multitemporal monitoring of variable infestation levels on water hyacinth plants. In "Seizing on sparsity in nonlinear hyperspectral unmixing for enhanced image compression" by A. Marinoni and P. Gamba, a strong detection of sparse distribution within the coefficients is embedded to enhance the performance of remotely sensed data compression. In "Endmember initialization method for hyperspectral data unmixing" by R. Wang et al., not only the true endmembers from the spectral library but also those close to the real hyperspectral images (HSI) are extracted, under the prior referred to as finiteness of the responding endmember to a mixed hyperspectral pixel and sparsity of the fractional abundance matrix. In "Super-resolution reconstruction of hyperspectral images using empirical mode decomposition and compressed sensing" by Z. Zhou, the spectral-spatial sparsity of HSI has been elaborately clarified to design a sparse representation reconstruction framework of HSI, which contains empirical mode decomposition, compressed sensing, and principal component analysis.

Finally, according to the sparse representation classification (SRC) paradigm, two papers further explore hyperspectral image pattern recognition. "Local-preserving sparse representation-based classification in hyperspectral imagery" by L. Gao et al. integrates local-preserving dimensional reduction and SRC to address the imbalance between high dimensionality of hyperspectral data and the limited number of training samples. "Change detection with one-class sparse representation classifier" by Q. Ran et al. incorporates the one-class strategy into SRC achieving more accurate representations of the change class, and proving the effectiveness of this option for data collected during the flood in the Heilongjiang Province, China, in 2013.

All of these papers show the maturity and extreme interest in the topic of this special section. They were selected thanks to the effort of a large number of reviewers, whose work and dedication are here deeply acknowledged.

Xin Huang is currently a full professor with Wuhan University, where he is the founder and director of the Institute of Remote Sensing Information Processing, School of Remote Sensing and Information Engineering. He has published more than 80 peer-reviewed articles in international journals. He was the recipient of the Boeing Award for the Best Paper in Image Analysis and Interpretation from the American Society for Photogrammetry and Remote Sensing in 2010, the National Excellent Doctoral Dissertation Award of China in 2012, and the China National Science Fund for Excellent Young Scholars in 2015. He was the winner of the IEEE GRSS 2014 Data Fusion Contest.

Paolo Gamba is professor of telecommunications at the University of Pavia, Italy, where he leads the Telecommunications and Remote Sensing Laboratory and serves as deputy coordinator of the PhD School in Electronics and Computer Science. He served as editor-in-chief of the IEEE Geoscience and Remote Sensing Letters from 2009 to 2013 and as chair of the Data Fusion Committee of the IEEE Geoscience and Remote Sensing Society from October 2005 to May 2009. He has been the organizer and technical chair of the biennial GRSS/ISPRS Joint Workshops on Remote Sensing and Data Fusion over Urban Areas since 2001. He has published more than 130 papers in international peer-reviewed journals and presented more than 250 research works in workshops and conferences.

Bormin Huang is the director of Intel Parallel Computing Center at the University of Wisconsin-Madison, a research scientist with the Space Science and Engineering Center at the University of Wisconsin-Madison, a research professor with the University of Las Palmas de Gran Canaria, an international chair professor with National Taipei University of Technology, a Thousand Talents Chair Professor at Xidian University and a guest professor at several universities in China. He has been the lead chair for the SPIE Conference on Satellite Data Compression, Communications, and Processing and for the SPIE Europe Conference on High-Performance Computing in Remote Sensing. He is a Fellow of SPIE and an NVIDIA CUDA Fellow. 